\title{
DOI:10.19122/201400000000000002
}

Capítulo 2

\section{Fibrogênese nas doenças inflamatórias intestinais}

\author{
Heitor S. P. de Souza \\ Claudio Fiocchi
}

\section{Introdução}

Fibrose resulta de lesão tecidual e pode ser tanto benéfica, quanto prejudicial ao organismo. Em geral, quando induzida em resposta a um insulto de intensidade leve e de curta duração, a fibrose constitui um processo fisiológico regulado, destinado à cicatrização e ao reparo da função do tecido ou órgão afetado. Por outro lado, quando a agressão é intensa e de natureza crônica, a resposta fibrótica consequente pode gerar anormalidades estruturais e funcionais no órgão afetado, eventualmente causando sintomas incapacitantes. Do ponto de vista celular, fibrose é definida como o resultado final de uma deposição excessiva de matriz extracelular (MEC), uma mistura acelular de vários componentes incluindo proteínas tais como o colágeno, fibronectina, proteoglicanos, entre outros. O processo subjacente à fibrose é chamado de fibrogênese, que tem sido reconhecido como sendo muito mais complexo do que se imaginava até pouco tempo, com uma variedade de causas e mediado por muitos tipos diferentes de células, vias de ativação, fatores de crescimento, citocinas e componentes da MEC. Nesta revisão, discutiremos os mecanismo gerais da fibrogênese, além daqueles relevantes para a inflamação intestinal e a doença inflamatória intestinal (DII) em particular, e apresentaremos conceitos atuais sobre prevenção e terapia para a fibrose intestinal. 


\section{Mecanismos básicos de fibrogênese}

A progressão básica de uma resposta fibrogênica inclui múltiplas fases que ocorrem sequencialmente, e em função do tempo. 0 processo começa com a lesão inicial, imediatamente seguida por uma fase de hemostasia, depois uma fase inflamatória e regenerativa, e uma fase final de maturação, em que sobrevém o remodelamento tecidual, culminado com o estabelecimento de fibrose ${ }^{1}$.

Dentre as muitas células que participam da fibrogênese, aquela que desempenha função central é a célula mesenquimal, presente basicamente em todos os órgãos em três formas distintas e inter-relacionadas: o fibroblasto, o miofibroblasto, e a célula muscular. Uma vez exposta à influência de células do sistema imunitário e seus produtos (primariamente citocinas e fatores de crescimento), a célula mesenquimal se transforma em miofibroblasto ativado, principal responsável pela produção e deposição de MEC. Esse processo de ativação está sob a influência de células do sistema imunitário inato e do adaptativo, incluindo neutrófilos, macrófagos e eosinófilos, além de linfócitos T auxiliares (Th)1, Th2, Th17, e linfócitos T regulatórios (Treg), respectivamente ${ }^{2}$. Todas essas células produzem uma ampla variedade de mediadores solúveis que agem sobre as células mesenquimais, promovendo a sua diferenciação, proliferação e migração, a síntese de MEC e, finalmente, a indução de miofibroblastos ativados ${ }^{3}$. Dentre os mediadores mencionados, o TGF- $\beta$ (transforming growth fator- $\beta$ ) é considerado o mais importante para a produção e a ativação de miofibroblastos, embora outros vários mediadores também possam contribuir, tais como a interleucina (IL)-1, IL-4, IL-6, IL-13 e IL-12, os fatores de crescimento do tecido conjuntivo (CTGF), o derivado de plaquetas (PDGF), o de fibroblasto (FGF), o epidérmico (EGF), o semelhante à insulina (IGF), e a osteopontina (OPN). Alguns dos mesmos mediadores derivados de células imunocompetentes exercem uma ação inibitória oposta, tais como IL-12, IL-10, interferon-gama (IFN)-g e o TGF- $\beta$, enquanto que outros parecem induzir tanto estimulação positiva quanto negativa, dependendo das condições experimentais, como é o caso do fator de necrose tumoral (TNF)- $\alpha^{3}$. Um conceito adicional relevante para a fibrogênese reside no fato de que as células mesenquimais não são ativadas exclusivamente em resposta a influências biológicas, 
tais como as descritas acima, mas também mecânicas, incluindo tensão, cisalhamento, compressão, pressão hidrostática e osmótica, força gravitacional, etc. ${ }^{4}$. Esses processos mecânico-biológicos são mais difíceis de estudar e de avaliar sua contribuição relativa à fibrose, mas é provável que eles assumam diferentes graus de relevância na dependência de que órgão ou tecido estaria sofrendo o estímulo fibrogênico.

\section{Fibrogênese na inflamação intestinal}

Em condições fisiológicas do sistema gastrointestinal, em estado de homeostase tecidual, todas as células locais se dividem e exercem suas ações, de forma a alcançar e manter o bom funcionamento do trato gastrointestinal. Frente à exposição a determinado estímulo, a resposta universal, básica, será de inflamação intestinal, a qual, dependendo do tipo, grau, e duração da agressão, pode resultar em regeneração ou reparo ${ }^{5}$. Em tecidos em constante renovação, como o epitélio gastrointestinal, uma lesão como uma úlcera péptica não complicada, pode evoluir para cura sem nenhuma cicatriz residual, isto é, regeneração completa. Em um tecido mais estável, com menos renovação, tal como as camadas musculares do trato gastrointestinal, uma lesão tal como a induzida por diverticulite crônica ou aguda, resultará em cicatriz localizada, isto é, a produção de fibrose localizada. Todavia, caso o músculo seja afetado de forma contínua e intensa, por exemplo, como na doença de Crohn (CD), é bastante provável que venha a se desenvolver uma fibrose mais difusa. Portanto, as causas, consequências e desfechos do processo fibrogênico no intestino podem, de fato, ser muito variáveis, assim como as suas implicações clínicas.

\section{Mecanismos de fibrogênese em DII}

As duas formas de DII, a DC e a retocolite ulcerativa (RCU), constituem entidades complexas e heterogêneas, apresentando manifestações clínicas variáveis, algumas das quais devidas ao processo primário de doença, ou seja, a inflamação, e algumas por conta de complicações secundárias, incluindo a fibrose intestinal. Os sintomas na DC, por exemplo, variam substancialmente de acordo com o curso da doença. Tal como o demonstrado pelo acompanhamento 
por período de dez anos no estudo de coortes IBSEN, os sintomas podem ser intensos no início e depois diminuir com o tempo (43\%), ou os pacientes podem apresentar sintomas crônicos intermitentes com períodos de remissão nos intervalos (32\%); de maneira alternativa sintomas crônicos contínuos podem persistir através do curso da doença (19\%), ou ainda serem mais leves no início, aumentando com o passar do tempo $(3 \%)^{6}$. Esses cursos imprevisíveis de doença dependem, em parte, da distribuição anatômica da doença (ileal, íleo-colônica, colônica, ou gastroduodenal), mas também do tipo de lesão sofrida pelo intestino, como, por exemplo, inflamação, fistulização, ou obstrução. Esses últimos três representam os subtipos principais de comportamentos na DC, mas esses fenótipos não são necessariamente estáveis ou estanques. De fato, o relato clássico de Cosnes e cols. ${ }^{7}$, que seguiram um grande número de pacientes durante mais de vinte anos, mostra que a maioria dos pacientes apresentam-se primariamente com inflamação isoladamente mas, com o passar do tempo, a proporção desses casos de fenótipo puramente inflamatório diminui, enquanto a proporção daqueles com fenótipos fistulizantes e obstrutivos aumenta.

O fenótipo obstrutivo surge em consequência de estenose progressiva da luz intestinal, primordialmente no intestino delgado, ocasionando o que se chama DC fibroestenosante, tipicamente exemplificada pelo clássico sinal de estreitamento de alça, principalmente no íleo terminal, observado em exames de imagem. A redução do lúmen na DC fibroestenosante constitui complicação da doença e deve-se ao espessamento das camadas do intestino, incluindo a mucosa, a submucosa, a muscularis propria e a serosa, um fenômeno secundário à inflamação crônica transmural. Essa sequência de eventos claramente indica que a fibrogênese na DC é consequência direta do processo inflamatório persistente característico dessa forma de DII. Essa série de eventos, contudo, não é exclusividade da DC. Entretanto, deve-se notar que, contradizendo o conhecimento popular de que fibrose ocorre somente na DC, vários graus de fibrose também podem ser encontrados na $\mathrm{RCU}^{8}$. A diferença, contudo, está na localização da resposta fibrótica. Pelo fato de a inflamação na RCU ser restrita à mucosa e à submucosa superficial, o processo 
fibrogênico segue um padrão semelhante: dependendo da intensidade da inflamação e de sua duração, pode-se encontrar a camada muscular da mucosa hipertrófica, com uma submucosa fibrótica espessa.

Os mecanismos moleculares e celulares responsáveis pelas alterações estruturais e funcionais mais proeminentes que o intestino sofre na DII são múltiplos e complexos. De uma forma geral, os mecanismos de fibrogênese na DIl são bastante similares aos descritos acima como mecanismos gerais de fibrogênese. Todavia, algumas diferenças substanciais podem ser observadas, estando relacionadas com a anatomia do órgão, sua composição celular, o tipo de conteúdo luminal, e a absorção de produtos microbianos e da dieta. Nos próximos parágrafos, serão enumerados e discutidos como cada um desses componentes podem contribuir para a fibrose na DII.

\section{Células mesenquimais}

Os miofibroblastos ativados também são considerados como o principal componente fibrogênico mesenquimal responsável pela fibrose intestinal associada à DII. Em condições normais, o intestino contém um grande número de células mesenquimais na forma de fibroblastos, miofibroblastos, e células musculares, cada uma delas com características únicas e compartilhadas, sendo que todos os três tipos podem dar origem a miofibroblastos ativados, produtores de $\mathrm{MEC}^{9}$. Acredita-se que esses três tipos de células constituam os principais mediadores da fibrose intestinal, mas certamente eles não são os únicos, pois existem evidências experimentais indicando que muitas outras células mesenquimais e não-mesenquimais possam também contribuir para a produção de MEC. Sob determinadas circunstâncias, primariamente relacionadas com uma pressão inflamatória intensa, as células epiteliais e endoteliais sofrem o processo de transição epitélio-mesenquimal (TEM) e endotélio-mesenquimal (TEndoM). Os pericitos de pequenos vasos têm sido alvo de intensa investigação, como importantes mediadores de fibrogênese. Fibrócitos, células produtoras de colágeno que se assemelham monócitos, podem ser recrutados da circulação sanguínea para sítios inflamatórios, e então se diferenciarem em células mesenquimais ${ }^{10}$, fato que pode ocorrer também com células-tronco derivadas da medula 
óssea. As células estreladas, o tipo celular primariamente responsável pela fibrose hepática, também podem ser encontradas na mucosa intestinal humana (Leite e cols., observações não publicadas) e exibem características típicas que incluem a presença de glóbulos de gordura e vitamina A, e se diferenciam in vitro em células típicas, positivas para $\alpha$-actina de músculo liso. Também é interessante notar que essa diferenciação é mais rápida quando as células estreladas derivam do intestino de pacientes com DII, uma indicação da provável existência de alguma forma de indução in vivo, no contexto do microambiente inflamatório das DII. Na mucosa, todas as células mesenquimais, independentemente de sua origem, estão expostas a uma variedade de fatores locais, interagindo tanto com células da imunidade inata, da imunidade adaptativa, como com células de origem não-imunitária, e os produtos secretados por todas elas. Essas interações têm características diferentes, de acordo as condições subjacentes, se fisiológicas ou inflamatórias, e determinam o destino final e a atividade pró-fibrótica das células mesenquimais.

\section{Matriz extracelular e enzimas proteolíticas}

Tal como referido anteriormente, a deposição excessiva de MEC constitui uma marca característica da fibrose intestinal. A MEC é constituída de muitos tipos diferentes de moléculas, todas produzidas por miofibroblastos ativados: colágenos (tipos I a VI), fibronectinas (EDA e EDB), elastinas, fibrilinas, proteínas matricelulares (CTGF, osteopontina, e SPARC), tenascinas (C e X), e proteoglicanos (hyaluronan, syndecam, HSPGs, e SLRPs) ${ }^{11}$. A MEC ocupa o espaço intercelular, ligando e permeando todas as células, mas não é uma estrutura inerte. Ao contrário, a MEC é biologicamente ativa e modula a ativação, migração, e a função geral das células mesenquimais, através das integrinas por elas expressadas ${ }^{12}$. Conforme o esperado, os níveis de vários componentes da MEC estão elevados nos tecidos afetados pela DII, particularmente dos colágenos tipo I, II e IV ${ }^{13,14}$.

A produção e a deposição de MEC não constitui um processo unidirecional. De fato, a matriz depositada sofre constante processo de modificação, no qual alguns componentes da MEC são degradados, enquanto que outros são substituídos. A degradação da MEC no intestino é mediada por um grande número de diferentes proteases 
de origem microbiana, epitelial, imunitária, e mesenquimal ${ }^{15}$. Na camada de muco, bactérias produzem colagenase A, fragilisina, hemaglutinina, gelatinase, e galactosidase; as células epiteliais produzem metaloproteinases da matriz (MMP)-1, MMP-2, MMP-7, e MMP-10; na lâmina própria, neutrófilos, macrófagos, e células mesenquimais produzem MMP-1, MMP-2, MMP-3, MMP-8, MMP-9, MMP-10, MMP12 e MMP-13. Todas essas enzimas têm atividades proteolíticas amplas, abrangentes, mas também específicas, podendo atuar sobre diversos substratos com resultados diferentes, dependendo do grau de inflamação e de fibrose concomitante. A atividade proteolítica das várias MMPs é contrabalançada por inibidores das MMPs, os TIMPs. Conforme o esperado, MMPs e outras enzimas proteolíticas apresentam níveis elevados tanto na DC como na RCU ${ }^{16}$.

\section{0 processo inflamatório}

A fibrose associada com DII é basicamente uma resposta secundária ao processo inflamatório crônico local. Esse processo envolve uma enorme quantidade de células imunitárias e outras de origem não-imunitária primariamente, ou não-linfóide, e uma variada gama de citocinas, fatores de crescimento, e mediadores solúveis. Portanto, o mecanismo que induz a fibrose na DII é basicamente similar ao que acontece em outas doenças que cursam com fibrose, mas a combinação de células e mediadores varia de doença a doença, e de tecido a tecido, de forma que algumas peculiaridades devem existir na fibrogênese associada com DC e RCU ${ }^{17}$.

Dentre os diversos fatores de crescimento promotores de fibrose na DII, o TGF- $\beta$ é considerado o mais poderoso. O TGF- $\beta$ é produzido de forma abundante por células do sistema imunitário inato, em particular por macrófagos, mas também pelas próprias células mesenquimais e, portanto, atuando de forma parácrina e autócrina. Os efeitos pró-fibróticos do TGF- $\beta$ são muitos, e incluem a atração e a ativação de células produtoras de MEC, assim como a promoção das transições TEM e TEndoM. $O$ efeito biológico do TGF- $\beta$ que contribui para as diversas doenças fibróticas está, não somente na síntese, mas também na quebra de proteínas da MEC, incluindo colágenos, fibronectinas, e proteoglicanos. As ações de moléculas da família do TGF- $\beta$ são mediadas através de três receptores, que 
ativam proteínas Smad, responsáveis pela transdução do sinal da superfície celular para o núcleo. O TGF- $\beta$ parece ser importante no remodelamento tecidual em DII, por influenciar o equilíbrio entre o aumento de produção e a deposição, além de uma degradação comprometida de componentes da MEC. Outros fatores de crescimento implicados na fibrogênese da DIl são as ativinas, membros da superfamília do TGF- $\beta$, CTGF, que atua a jusante do TGF- $\beta$, PDGF, IGFs, $E G F$, endotelinas, e membros do sistema renina-angiotensina, todos esses encontram-se alterados no tecido de pacientes com DII ${ }^{17}$.

Anormalidades em células Th1, Th2, Th17, e em Tregs, são reconhecidas como fundamentais não somente para a patogênese das DIls, mas também para o processo fibrogênico associado. Isso se dá, primariamente, através da produção local de citocinas respectivamente à resposta Th, incluindo IL-4, IL-6, IL-13, IL-17, IL-21, IL-22, IL-23, IL-33 e TNF- $\alpha^{18}$. As ações dessas citocinas são distintas e sua contribuição relativa a fibrogênese ainda esta sob investigação. As citocinas IL-4 e IL-13 são consideradas como algumas das citocinas pró-fibróticas mais potentes, induzindo a ativação e a diferenciação de fibroblastos para miofibroblastos, e a produção de colágenos ${ }^{18}$. O eixo IL-23/IL-17 é considerado como mediador chave para a DC, e a IL-17 tem sido implicada na ativação de miofibroblastos intestinais $^{19}$. IL-1 e IL-33 são membros da superfamília da IL-1 de citocinas, e ambas estão elevadas nas DIls, tendo a capacidade de promover fibrogênese. IL-6 e TNF- $\alpha$ são citocinas pró-inflamatórias potentes, produzidas de forma abundante nas Dlls, mas sua ação pró-fibrótica em DC e RCU ainda não está bem definida.

\section{A microbiota intestinal}

Um aspecto que estabelece uma diferença dramática entre o intestino e outros órgãos e tecidos do corpo, está na presença de uma rica microbiota na luz intestinal, em íntima associação com a mucosa. A microbiota intestinal tem sido reconhecida atualmente como fundamental à homeostase imunitária do intestino, e é considerada como alvo da resposta imunitária anormal subjacente à patogênese das $\mathrm{DIls}^{20}$. Apenas recentemente, contudo, a conexão entre a microbiota intestinal e a fibrogênese começou a ser explorada nas DIls, especialmente considerando que as células mesenquimais da mucosa - e os miofibroblastos 
sub-epiteliais, em particular - são separadas por apenas uma camada de células epiteliais, da luz intestinal com sua microbiota. Portanto, qualquer quebra da barreira epitelial acabará por expor as células mesenquimais locais a uma variedade de produtos bacterianos que serão reconhecidos através dos Toll-like receptors (TLR) e dos NOD-like receptors expressos em células mesenquimais. Estudos em curso em nosso laboratório têm mostrado que os fibroblastos intestinais humanos são ativados seletivamente por TLR5, cujo ligante único é a flagelina, um antígeno presente exclusivamente em bactérias flageladas. 0 resultado desse processo de ativação seletivo é o aumento de produção de colágeno e fibronectina, uma resposta que estabelece uma ligação nova e direta da microbiota intestinal com a fibrogênese nas Dlls. Uma vez que, nas DIls, a barreira mucosa está comprometida secundariamente à lesão inflamatória, o acesso de produtos bacterianos à mucosa torna-se constante, e isso poderia explicar como a microbiota pode contribuir para a fibrose intestinal, tanto na DC quanto na RCU. Com isso, vem à tona outra questão conjectural sobre se existiria uma microbiota pró-fibrogênica em alguns pacientes com DII, particularmente suscetíveis à fibrose. Poder-se-ia imaginar que três tipos de microbiota devam existir: uma normal, que mantém a homeostasia da mucosa, uma microbiota pró-inflamatória, que promove excessiva reatividade imunitária e inflamação, e uma pró-fibrogênica, que induz seletivamente fibrose. Essa ideia é sustentada por evidências de que a presença de microbiotas diferentes em tecidos lesionados podem levar à cicatrização, ou à persistência da lesão e fibrose ${ }^{21}$, o que implicaria que a modulação da microbiota poderia ter valor terapêutico na prevenção ou resolução da fibrose intestinal.

\section{Fatores ambientais}

Todo o conteúdo presente na luz intestinal deve ser considerado como uma combinação de fatores ambientais, com o potencial de impactar a imunidade intestinal, a inflamação e a fibrose. Assim como a microbiota, discutida na seção anterior, os produtos da dieta e seus metabólitos também são componentes do conteúdo intestinal e, portanto, participantes dessas interações. Recentemente, evidências têm se acumulado no sentido de que a composição da microbiota intestinal é diretamente e significativamente dependente da dieta ${ }^{22}$. 
Dentre os muitos componentes dietéticos, a gordura pode promover a inflamação intestinal por estimular o fluxo linfático, induzir a liberação de citocinas por macrófagos, linfócitos intraepiteliais e enterócitos, a ativação de mastócitos e, consequentemente influenciando a função imunitária ${ }^{23}$. Evidências adicionais do potencial pró-inflamatório e pró-fibrótico da gordura advém de resultados de estudos in vivo mostrando que ovelhas com obesidade induzida por gordura, ao contrário de ovelhas magras, dão à luz recém-natos com inflamação intestinal e fibrose ${ }^{24}$. Baseado nessas observações, o volume aumentado de gordura mesentérica e daquela que envolve a alça, característicos da DC, deveriam ser vistos provavelmente como possíveis contribuintes da fibrose intestinal ${ }^{25}$. Estudos em curso em nosso laboratório, oferecem sustentação para essa possibilidade, tal como o sugerido por achados de que alguns ácidos graxos livres promovem a proliferação e a migração de fibroblastos intestinais humanos, talvez estabelecendo uma ligação fisiopatológica que explicaria a observação de co-localização da gordura envolvente, com segmentos de fibroestenose na DC.

\section{Transformação celular}

Além da ação direta da inflamação sobre a função das células mesenquimais e a estimulação da fibrose, a inflamação também pode atuar através da transformação de alguns tipos celulares não imunitários em unidades mesenquimais, do ponto de vista morfológico e funcional. Existe também boa base de sustentação indicando que a inflamação, dependendo da intensidade e da persistência, pode realmente induzir a transformação de células epiteliais e endoteliais em células mesenquimais, primariamente através da ação de citocinas (TGF- $\beta$, IL-1 $\beta$, TNF- $\alpha$ ), fatores de crescimento, fibrinogênio, e MMPs ${ }^{26}$. Isto também pode ocorrer no intestino inflamado, tal como o indicado em relatos de TEM em DII experimental, e de TEndoM em DII humana e experimental27,28. Até que ponto transformações celulares contribuem para a fibrogênese nas Dlls é difícil de quantificar, mas a importância dessas observações reside na extraordinária capacidade da inflamação de promover respostas fibróticas através de uma variedade de mecanismos diferentes. Essa potencialidade é mediada por modificações epigenéticas, tal como o mostrado por um aumento progressivo na expressão do gene colágeno IA2 por células endote- 
liais sob a influência de citocinas produzidas na mucosa inflamada de DIl, tais como IL-1 $\beta$, TGF- $\beta$ e TNF- $\alpha^{29}$. Embora ainda não investigadas, modificações epigenéticas provavelmente também ocorrem na TEM associada com a formação de fístula em paciente com $\mathrm{DC}^{30}$. Outro mecanismo de modificação epigenética relevante a fibrose, é aquele mediado por microRNAs (miRNA) ${ }^{31}$, mas relatos de anormalidades na expressão de miRNA ligados à fibrogênese em DII ainda carecem de base na literatura científica.

\section{Prevenção e terapia da fibrose intestinal}

Apesar de os recentes avanços na compreensão de eventos subjacentes à fibrogênese em DII, um manejo clínico efetivo da fibrose na DC ou na RCU, continua fora do alcance nos dias de hoje. Nesse sentido, duas questões principais precisam ser levadas em consideração: em primeiro lugar, será que a fibrose intestinal pode ser prevenida e, em segundo, uma vez estabelecida, será que a fibrose pode ser reduzida ou revertida? 0 uso de agentes imunossupressores e dos biológicos durante as últimas décadas têm tido um grande impacto positivo sobre o controle da inflamação e da qualidade de vida tanto em pacientes com DC quanto naqueles com RCU, mas aparentemente não preveniu complicações, tais como a formação de estenoses, a obstrução intestinal e a necessidade de cirurgia ${ }^{32}$.

Em relação à prevenção da fibrose intestinal, ainda não existem abordagens práticas sensíveis o suficiente para detectá-las nos seus primórdios, ainda que novas técnicas de exames de imagem tenham surgido e algumas ainda estejam em desenvolvimento ${ }^{33}$. Entretanto, no panorama experimental, existem ainda evidências limitadas, mas intrigantes, de que a fibrose se desenvolveria precocemente, em paralelo à inflamação, tal como na colite experimental induzida pela infecção por salmonela ${ }^{35}$. No referido modelo, se o agente agressor for eliminado cedo no curso da infecção, a fibrose regride; no entanto, se eliminado mais tardiamente e a inflamação perdurar por mais tempo, a fibrose torna-se persistente, mesmo após a erradicação do agente agressor. Esta importante observação apoia de forma consistente a ideia de que a fibrose pode ser prevenida, contudo, somente quando detectada precocemente e sua causa completamente removida, algo que, no momento, não pode ser alcançado em pacientes com DII. Va- 
riantes genéticas associadas com fibrose têm sido relatadas em fibrose hepática e na esquistossomose, mas elas não foram detectadas em associação com DII, embora algumas variantes de colágeno tenham sido descritas recentemente ${ }^{35} \mathrm{cH}$. Portanto, no momento, não é possível identificar indivíduos com Dll que tenham maior risco para o desenvolvimento de fibrose intestinal. Outro dado novo interessante, é que uma vez estabelecida a fibrose, a própria rigidez do segmento intestinal acometido parece promover ainda mais fibrose, através ativação e indução de fibroblastos intestinais, que passam a produzir mais $\mathrm{MEC}^{36}$. Esse tipo de resposta cria um ciclo vicioso, mantendo e exacerbando a fibrogênese intestinal. Recentemente, algumas novas medicações como o CCG-100602, que bloqueia a sinalização fibrogênica derivada da rigidez, estão sendo testadas com o intuito de interromper esse mecanismo de autoperpetuação ${ }^{37}$.

A fibrose tem sido tradicionalmente considerada como irreversível na maioria das situações clínicas, incluindo as DIls, mas de fato, mesmo os fibroblastos, as principais células produtoras de MEC, podem ser convertidos em outras células não-produtoras de $\mathrm{MEC}^{38}$, o que sugere que a fibrose possa ser teoricamente reversível mesmo após estar bem estabelecida. A indústria farmacêutica está desenvolvendo um grande número de novas estratégias anti-fibróticas baseadas em diversas abordagens tais como o bloqueio de fatores de crescimento, anti-integrinas, enzimas intracelulares, moléculas anti-inflamatórias, antioxidantes, modificadores da coagulação entre outros ${ }^{2}$. Portanto, as bases teóricas para alterar a fibrose na maioria dos órgãos encontram-se em diferentes fases clínicas, e alguns ensaios clínicos estão em curso no momento, sendo alguns deles dirigidos à DC (Embase, Medline, www.clinicaltrials.gov). Entretanto, importantes obstáculos ainda precisam ser ultrapassados antes que agentes efetivos e práticos, especificamente orientados ao tratamento da fibrose intestinal possam se tornar disponíveis ${ }^{39}$. Esses obstáculos incluem a natureza e o momento do estímulo agressor inicial, a lenta progressão do processo fibrótico, a ausência de biomarcadores validados, e parâmetros clínicos mal definidos; tais obstáculos, por sua vez, determinam uma longa duração dos ensaios clínicos, a necessidade de um grande número de pacientes, e o elevado custo das pesquisas clínicas. 


\section{Referências Bibliograficas}

1. Wynn TA. Fibrotic disease and the $T(H) 1 / T(H) 2$ paradigm. Nat Rev Immunol. 2004 Aug;4(8):58394. PubMed PMID: 15286725. Pubmed Central PMCID: 2702150. Epub 2004/08/03.

2. Wynn TA, Ramalingam TR. Mechanisms of fibrosis: therapeutic translation for fibrotic disease. Nat Med. 2012;18(7):1028-40. PubMed PMID: 22772564. Epub 2012/07/10.

3. Wick G, Backovic A, Rabensteiner E, Plank N, Schwentner C, Sgonc R. The immunology of fibrosis: innate and adaptive responses. Trends Immunol. 2010 Mar;31(3):110-9. PubMed PMID: 20106721. Epub 2010/01/29. eng.

4. Huang C, Ogawa R. Fibroproliferative disorders and their mechanobiology. Connect Tissue Res. 2012;53(3):187-96. PubMed PMID: 22329637. Epub 2012/02/15.

5. Fiocchi C, Lund PK. Themes in fibrosis and gastrointestinal inflammation. Am J Physiol Gastrointest Liver Physiol. 2011 Mar 17. PubMed PMID: 21415411. Epub 2011/03/19.

6. Solberg IC, Lygren I, Jahnsen J, Aadland E, Høie O, Cvancarova M, et al. Clinical course during the first 10 years of ulcerative colitis: results from a population-based inception cohort (IBSEN Study). Scand J Gastroenterol. 2009;44(4):431-40.

7. Cosnes J, Cattan S, Blain A, Beaugerie L, Carbonnel F, Parc R, et al. Long-term evolution of disease behavior of Crohn's disease. Inflamm Bowel Dis. 2002 Jul;8(4):244-50. PubMed PMID: 12131607. Epub 2002/07/20.

8. Gordon IO, Agrawal N, Goldblum JR, Fiocchi C, Rieder F. Fibrosis in Ulcerative Colitis: Mechanisms, Features, and Consequences of a Neglected Problem. Inflamm Bowel Dis. 2014 Jun 2. PubMed PMID: 24892966. Epub 2014/06/04.

9. Rieder F, Fiocchi C. Intestinal fibrosis in IBD--a dynamic, multifactorial process. Nat Rev Gastroenterol Hepatol. 2009 Apr;6(4):228-35. PubMed PMID: 19347014. Epub 2009/04/07.

10. Peng H, Herzog EL. Fibrocytes: emerging effector cells in chronic inflammation. Curr Opin Pharmacol. 2012 Aug;12(4):491-6. PubMed PMID: 22465542. Pubmed Central PMCID: 3651702. Epub 2012/04/03.

11. Klingberg F, Hinz B, White ES. The myofibroblast matrix: implications for tissue repair and fibrosis. J Pathol. 2013 Jan;229(2):298-309. PubMed PMID: 22996908. Pubmed Central PMCID: 4005341. Epub 2012/09/22.

12. Leeb SN, VogI D, Gunckel M, Kiessling S, Falk W, Goke M, et al. Reduced migration of fibroblasts in inflammatory bowel disease: role of inflammatory mediators and focal adhesion kinase. Gastroenterology. 2003 Nov;125(5):1341-54. PubMed PMID: 14598250. Epub 2003/11/05.

13. Stallmach A, Schuppan D, Riese HH, Matthes H, Rieken EO. Increased collagen type III synthesis by fibroblasts isolated from strictures of patients with Crohn's disease. Gastroenterology. 1992;102:1920-9.

14. Matthes H, Herbst H, Schuppan D, Stallmach A, Milani S, Stein H, et al. Cellular localization of procollagen gene transcripts in inflammatory bowel disease. Gastroenterology. 1992;102:43142.

15. Biancheri P, Di Sabatino A, Corazza GR, MacDonald TT. Proteases and the gut barrier. Cell Tissue Res. 2013 Feb;351(2):269-80. PubMed PMID: 22427120. Epub 2012/03/20.

16. Baugh MD, Perry MJ, Hollander AP, Davies DR, Cross SS, Lobo AJ, et al. Matrix metalloproteinases levels are elevated in inflammatory bowel disease. Gastroenterology. 1999;117:814-22.

17. Speca S, Giusti I, Rieder F, Latella G. Cellular and molecular mechanisms of intestinal fibrosis. World J Gastroenterol. 2012 Jul 28;18(28):3635-61. PubMed PMID: 22851857. Pubmed Central PMCID: 3406417. Epub 2012/08/02.

18. Borthwick LA, Wynn TA, Fisher AJ. Cytokine mediated tissue fibrosis. Biochim Biophys Acta. 2013 Jul;1832(7):1049-60. PubMed PMID: 23046809. Pubmed Central PMCID: 3787896. Epub 2012/10/11.

19. Hata K, Andoh A, Shimada M, Fujino S, Bamba S, Araki Y, et al. IL-17 stimulates inflammatory responses via NF-KB and MAP kinase pathways in human colonic myofibroblasts. Am J Physiol. 2002;282:G1035-G44.

20. Kostic AD, Xavier RJ, Gevers D. The microbiome in inflammatory bowel disease: current status and the future ahead. Gastroenterology. 2014 May;146(6):1489-99. PubMed PMID: 24560869. Pubmed Central PMCID: 4034132. Epub 2014/02/25.

21. Scales BS, Huffnagle GB. The microbiome in wound repair and tissue fibrosis. J Pathol. 2013 
Jan;229(2):323-31. PubMed PMID: 23042513. Pubmed Central PMCID: 3631561. Epub 2012/10/09.

22. Wu GD, Chen J, Hoffmann C, Bittinger K, Chen YY, Keilbaugh SA, et al. Linking long-term dietary patterns with gut microbial enterotypes. Science. 2011 Oct 7;334(6052):105-8. PubMed PMID: 21885731. Epub 2011/09/03.

23. Ji Y, Sakata Y, Tso P. Nutrient-induced inflammation in the intestine. Curr Opin Clin Nutr Metab Care. 2011 Jul;14(4):315-21. PubMed PMID: 21587069. Epub 2011/05/19.

24. Yan X, Huang Y, Wang H, Du M, Hess BW, Ford SP, et al. Maternal obesity induces sustained inflammation in both fetal and offspring large intestine of sheep. Inflamm Bowel Dis. 2011 Jul;17(7):1513-22. PubMed PMID: 21674707. Epub 2011/06/16.

25. Desreumaux P, Ernst O, Geboes K, Gambiez L, Berrebi D, Muller-Alouf H, et al. Inflammatory alterations in mesenteric adipose tissue in Crohn's disease. Gastroenterology. 1999 Jul;117(1):73-81. PubMed PMID: 10381912. Epub 1999/06/26.

26. Kalluri R, Neilson EG. Epithelial-mesenchymal transition and its implications for fibrosis. J Clin Invest. 2003;112(12):1776-84.

27. Flier SN, Tanjore H, Kokkotou EG, Sugimoto H, Zeisberg M, Kalluri R. Identification of epithelial to mesenchymal transition as a novel source of fibroblasts in intestinal fibrosis. J Biol Chem. 2010;285(26):20202-12.

28. Rieder F, Kessler SP, West GA, Bhilocha S, de la Motte C, Sadler TM, et al. Inflammation-Induced Endothelial-to-Mesenchymal Transition: A Novel Mechanism of Intestinal Fibrosis. Am J Pathol. 2011 Sep 20. PubMed PMID: 21945322. Epub 2011/09/29.

29. Sadler T, Scarpa M, Rieder F, West G, Stylianou E. Cytokine-induced chromatin modifications of the type I collagen alpha 2 gene during intestinal endothelial-to-mesenchymal transition. Inflamm Bowel Dis. 2013 Jun;19(7):1354-64. PubMed PMID: 23635716. Pubmed Central PMCID: 3684204. Epub 2013/05/03.

30. Bataille F, Rohrmeier C, Bates R, Weber A, Rieder F, Brenmoehl J, et al. Evidence for a role of epithelial mesenchymal transition during pathogenesis of fistulae in Crohn's disease. Inflamm Bowel Dis. 2008;14(11):1514-27.

31. Bowen T, Jenkins RH, Fraser DJ. MicroRNAs, transforming growth factor beta- 1 , and tissue fibrosis. J Pathol. 2013 Jan;229(2):274-85. PubMed PMID: 23042530. Epub 2012/10/09.

32. Rieder F, Zimmermann EM, Remzi FH, Sandborn WJ. Crohn's disease complicated by strictures: a systematic review. Gut. 2013 Apr 26. PubMed PMID: 23626373. Epub 2013/04/30.

33. Zimmermann EM, Al-Hawary MM. MRI of the small bowel in patients with Crohn's disease. Curr Opin Gastroenterol. 2011 Mar;27(2):132-8. PubMed PMID: 21297471. Pubmed Central PMCID: 3433225. Epub 2011/02/08.

34. Johnson LA, Luke A, Sauder K, Moons DS, Horowitz JC, Higgins PD. Intestinal fibrosis is reduced by early elimination of inflammation in a mouse model of IBD: impact of a "Top-Down" approach to intestinal fibrosis in mice. Inflamm Bowel Dis. 2012 Mar;18(3):460-71. PubMed PMID: 21761511. Pubmed Central PMCID: 3206985. Epub 2011/07/16.

35. Elding H, Lau W, Swallow DM, Maniatis N. Refinement in localization and identification of gene regions associated with Crohn disease. Am J Hum Genet. 2013 Jan 10;92(1):107-13. PubMed PMID: 23246291. Pubmed Central PMCID: 3542460. Epub 2012/12/19.

36. Johnson LA, Rodansky ES, Sauder KL, Horowitz JC, Mih JD, Tschumperlin DJ, et al. Matrix stiffness corresponding to strictured bowel induces a fibrogenic response in human colonic fibroblasts. Inflamm Bowel Dis. 2013 Apr;19(5):891-903. PubMed PMID: 23502354. Pubmed Central PMCID: 3766259. Epub 2013/03/19.

37. Johnson LA, Rodansky ES, Haak AJ, Larsen SD, Neubig RR, Higgins PD. Novel Rho/MRTF/ SRF inhibitors block matrix-stiffness and TGF-beta-induced fibrogenesis in human colonic myofibroblasts. Inflamm Bowel Dis. 2014 Jan;20(1):154-65. PubMed PMID: 24280883. Epub 2013/11/28.

38. Szabo E, RampalliS, Risueno RM, Schnerch A, Mitchell R, Fiebig-Comyn A, etal. Direct conversion of human fibroblasts to multilineage blood progenitors. Nature. 2010 Nov 25;468(7323):5216. PubMed PMID: 21057492. Epub 2010/11/09.

39. Rieder $F$. The gut microbiome in intestinal fibrosis: environmental protector or provocateur? Sci Transl Med. 2013 Jun 19;5(190):190ps10. PubMed PMID: 23785034. Pubmed C 